\title{
Una metáfora no percibida en Antoninus Liberalis
}

Recibido: 2/07/2014

Aceptado: 1/09/2014

\section{RESUMEN:}

Se explica un pasaje de Antoninus Liberalis que no había sido entendido por los estudiosos.

PALABRAS CLAVE: Crítica textual; Antoninus Liberalis; Griego.

\section{ABSTRACT:}

A passage of Antoninus Liberalis which has been misunderstood by the critics is explained.

KEY WORDS: Textual criticism; Antoninus Liberalis; Greek language.

En mi artículo "Notas filológicas al texto de Antoninus Liberalis", que aparecerá en Myrtia, he señalado de paso que en Ant. Lib., Synagog. XXIV,3 la lectura $\mu \varepsilon \tau \alpha \lambda \alpha \beta \omega$ v es sana. Quisiera ahora explicar el pasaje en cuestión de manera exhaustiva, por medio de un análisis filológico del texto.

Es consabido que un verbo (participio o forma conjugada) cuando sea empleado en un sentido metafórico y no literal, puede ser

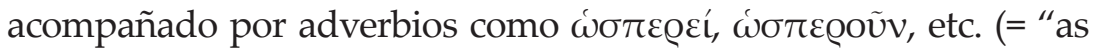
it were", "just as if", "por así decirlo": cf. e.g. Ael. NA IV,13 


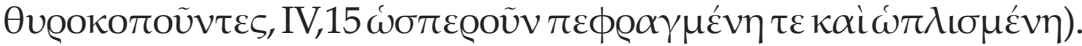
Pero dichos adverbios pueden ser omitidos por el escritor, en cuyo caso muchos críticos no comprenden la metáfora expresada por el verbo, y estropean el texto sugiriendo conjeturas infundadas. He indicado un ejemplo de tal omisión en Ant. Lib. Synagog. XII,4, donde $\varepsilon \chi \alpha \varrho \alpha \kappa \omega \dot{\theta} \theta \eta^{1}$ quiere decir -como los sabios no captaron- "fue, por decirlo así, circunvalado", o sea, remota metaphora, "fue inmovilizado"; me alegro mucho de ver que mis colegas J. Almirall i Sardà y E. Calderón Dorda, en su reciente edición de Antoninus Liberalis²,

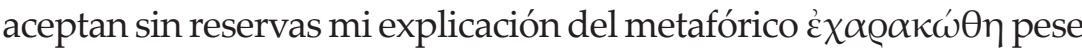
a que -lo que me sorprende- no se molestan en citarme.

Ahora bien, una análoga metáfora, que los estudiosos no han comprendido, ocurre en Ant. Lib. Synagog. XXIV,2-3, donde leemos que un joven, el $\pi \alpha \tilde{\imath} \varsigma$... $\tau \tilde{\eta} \varsigma$ Míon $\varsigma$, se burla de la diosa Deméter que está bebiendo demasiado ávidamente el $\kappa v \kappa \varepsilon \tilde{\omega}, \mathrm{y}$

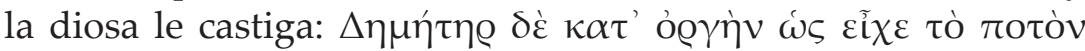

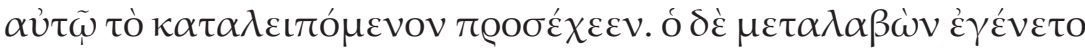

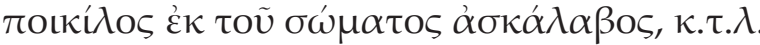

Este episodio es narrado por Ovidio, en Met. V,452 ss., en clave literal:

cum liquido mixta perfudit diva polenta:

combibit os maculas...

La diosa empapó (perfudit) la cara (os) del $\pi \alpha \tilde{i} \varsigma$ en la mixtura de agua y cebada, y la cara (os) de éste embebió (es decir, absorbió: combibit) las manchas (maculas) representadas por los granos de la cebada que se habían pegado a la piel de la faz.

Al contrario, en Antoninus Liberalis el episodio es visualizado en clave metafórica, o sea -patentemente, lo que mos-

1 Cf. Athlon. Satura grammatica in honorem F. R. Adrados, Madrid, 1987, p.368.

2 Antoní Liberal, Recull de metamorfosis, Barcelona, 2012, p. 150, n. 119. 


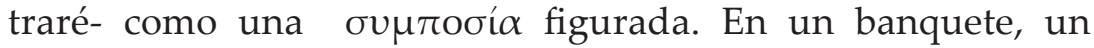

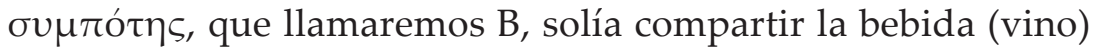

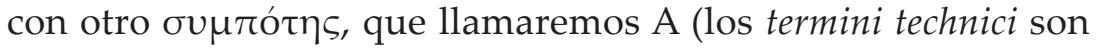

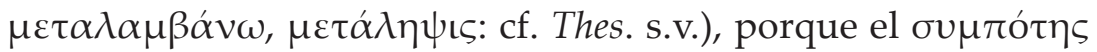
A ante todo bebía una parte del rotóv, y después pasaba el

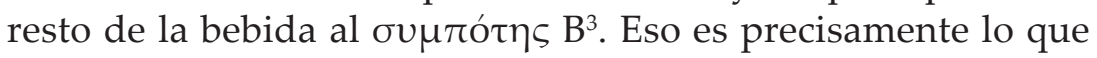
hace la diosa: después de haber bebido una parte del тotóv, ella echa el resto de la bebida en cara al $\pi \alpha \tilde{i} \varsigma^{4}$ que, metafóricamente, por así decirlo compartió la bebida con Deméter. El participio (metafórico) de aoristo $\mu \varepsilon \tau \alpha \lambda \alpha \beta \omega \nu v$ empleado sin $\omega ́ \sigma \pi \varepsilon \varrho \varepsilon i ́ ~ o ~ \omega ́ \sigma \pi \varepsilon \varrho o \tilde{v ~(=~ " p o r ~ a s i ́ ~ d e c i r l o, ~ h a b i e n d o ~ c o m p a r t i-~}$ do") es explicativo ${ }^{5}$, o sea, explica que el $\pi \alpha \tilde{\text { I}} \varsigma$ se transformó

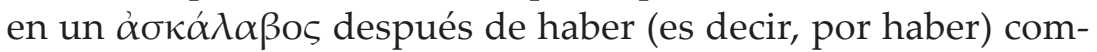

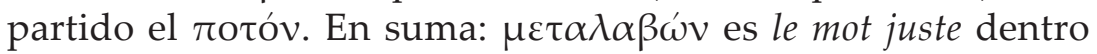
del marco de la $\sigma u \mu \pi$ rí́ $\alpha$ metafórica sobre la cual he echado luz. Sólo porque no podían comprender el valor metafórico del participio $\mu \varepsilon \tau \alpha \lambda \alpha \beta \omega ́ v$, los críticos se vieron constreñidos a estropearlo conjeturalmente, recurriendo a $\mu \varepsilon \tau \alpha \beta \alpha \lambda \omega \nu$ porque la frase $\mu \varepsilon \tau \alpha \beta \alpha \lambda \omega \nu$ غ่ $\gamma \varepsilon \dot{v} \varepsilon \tau$ o se lee en Ant. Lib. XI,10; XX,7; XXXII,2; y XXXVIII,5. Este estropicio conjetural es sobremanera infeliz, porque destruye la precisa y patente alusión que Antoninus Liberalis, como hemos visto, hace al susodicho "Greek and Roman custom" simpótico ${ }^{6}$. Está por esclarecer que la frase

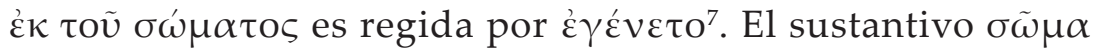
significa "cuerpo humano" en Ant. Lib. I,5; VII,5; XIII,2;

3 Cf. A Lexicon Abridged from Liddell and Scott's Greek-English Lexicon, Oxford, 1966, s.v. $\pi \varrho 0 \pi i v \omega:$ "the Greek, as also the Roman, custom was to drink first oneself, and then pass the cup to the person pledged".

4 El verbo $\pi \varrho o \sigma \chi \varepsilon ́ \omega$ significa "pour on...", cf. LSJ s.v.

5 Para este tipo de participios, cf. Kühner-Gerth II, p. 77 ss.

6 Cf. supra n. 3.

7 Para $\gamma^{\prime} \gamma^{\prime} \nu \mu_{\alpha} \alpha \dot{\varepsilon} \kappa+$ subst. denotando una transformación de algo en otra cosa,

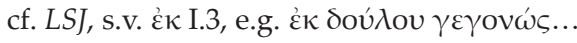




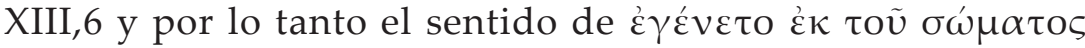
$\dot{\alpha} \sigma \kappa \alpha ́ \lambda \alpha \beta$ o podría ser "de su cuerpo humano llegó a ser un $\alpha \sigma \kappa \alpha \dot{\alpha} \alpha \beta \varsigma_{\text {o }}$ "; pero, dado que $\sigma \tilde{\omega} \mu \alpha$ puede significar $\pi \alpha \tilde{\mathrm{i}}$, y pues la Selbstvariation semántica (o sea, el empleo del mismo sustantivo en significados diversos) es de rigueur en los Aticistas $^{8}$, es probable que Antoninus Liberalis haya aquí em-

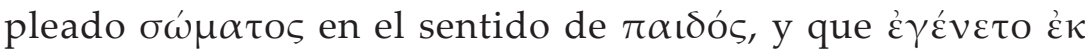

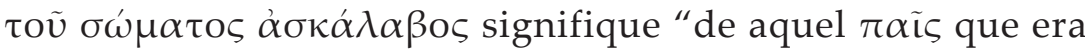
llegó a ser un $\alpha \dot{\sigma} \sigma \alpha \alpha \lambda \alpha \beta \varsigma^{9 \prime \prime}$.

Para concluir. La frase que he analizado (ó $\delta \dot{\varepsilon} \mu \varepsilon \tau \alpha \lambda \alpha \beta \omega \dot{v}$

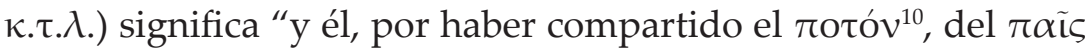

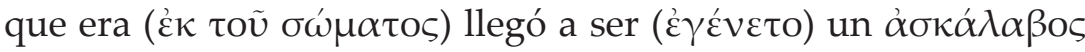
moteado". Nótese el hipérbaton, muy común en los Aticistas ${ }^{11}$ : el

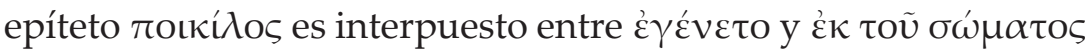
porque es enfático ${ }^{12}$, en tanto que pone de relieve el carácter notoriamente etiológico de la leyenda concerniente a la transfor-

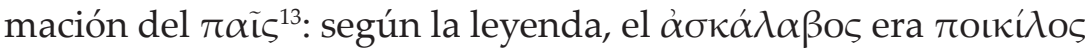
como consecuencia de las maculae epidérmicas producidas por el тoтóv.

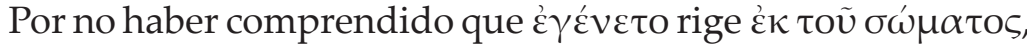
Almirall i Sardà y Calderón Dorda (op. cit.) construyen el texto de

8 Cf. MPhL 2002, p. 95.

9 La misma Selbstvariation en el empleo de $\sigma \tilde{\omega} \mu \alpha$ se encuentra en Eurípides, como he demostrado en Myrtia 2003, p. 31.

10 Por supuesto el pronombre $\alpha$ v่toṽ es sobreentendido: la omisión de los pronombres es habitual, cf. mi artículo citado arriba en n. 1, p. 363, y mi discusión de Ant. Lib. Synagog. XLI en mi susodicho artículo "Notas filológicas al texto de Antoninus Liberalis".

11 Cf. MPhL 2002, p. 95.

12 Cf. la nota 2 en mi artículo "Nesselrath Minervam" que aparecerá en Myrtia

13 Cf. la nota 235 en la edición de Antoninus Liberalis publicada por mis colegas Almirall i Sardà y Calderón Dorda citada en n. 2. 
una manera gramaticalmente errónea, traduciendo "el noi, camviant de forma, es convertí en dragó de cos pigallat": es un error

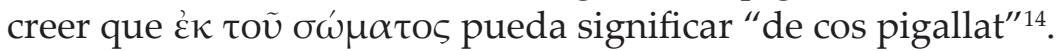

Giuseppe Giangrande

Professor EMERITUS UNIVERSITY OF LONDON

14 Nótese que, mientras que el participio explicativo $\mu \varepsilon \tau \alpha \lambda \alpha \beta \omega v^{\prime}$ "por haber compartido" es, como hemos visto, contextualmente apropiado, $\mu \varepsilon \tau \alpha \beta \alpha \lambda \omega \nu$ es abyectamente pleonástico, porque el sentido que produce sería "y él, transformándose, se transformó del $\pi \alpha \tilde{i} \varsigma$ que era en un $\alpha \sigma \kappa \kappa \alpha ́ \lambda \alpha \beta o \zeta$. 
